\title{
Ureteral Fibroepithelial Polyp
}

\author{
Zhen J. Wang, MD, Maxwell V. Meng, MD, Benjamin M. Yeh, MD, and Ruth B. Goldstein, MD \\ Department of Radiology (ZJW, BMY, RBG) and Department of Urology (MVM) University of \\ California San Francisco, 505 Parnassus Avenue, San Francisco, CA 94143-0628
}

Fibroepithelial polyps of the ureter are a rare benign neoplasm that can mimic other ureteral pathologies and lead to inaccurate diagnosis and treatment. The sonographic features of a vermiform and mobile solid ureteral mass may be suggestive of the diagnosis. We report a case of distal ureteral fibroepithelial polyp incidentally discovered at pelvic sonography in a young woman.

\section{Case Report}

A 39-year-old woman with a history of irregular menstrual bleeding was referred for pelvic sonogram for evaluation of possible uterine polyps. The patient reported occasional colicky right flank pain.

Pelvic sonogram showed an incidental vermiform solid mass in the urinary bladder near the right ureterovesical orifice (Figure 1). The mass was mobile, and appeared to arise from the right distal ureter, with portion of the mass protruding into the urinary bladder. A small amount of color flow was detected in the mass with color Doppler imaging. While the predominant mass was detected within the bladder, its exophytic and vermiform shape were felt to be atypical for a primary bladder tumor. No focal bladder wall thickening or hydroureteronephrosis was seen on sonography. The uterus and adnexa were normal.

The patient subsequently underwent CT urography for further evaluation of the ureteral and bladder mass. CT showed an elongated filling defect with a corkscrew appearance within the distal one third of the right ureter, with the mass protruding into the bladder (Figure 2). The filling defect measured at least $8 \mathrm{~cm}$ in length with smooth margin, and showed mild enhancement. On the CT, there was mild right hydronephrosis.

Both the sonographic and CT findings were consistent with a ureteral fibroepithelial polyp. The patient subsequently underwent cystoscopy and ureteroscopy which confirmed the presence of a right distal ureteral polyp. The polyp was ureteroscopically excised intact (Figure 3 ), and histology confirmed a fibroepithelial polyp.

\section{Discussion}

Ureteral fibroepithelial polyps are rare benign tumors that most frequently occur in adults but may also arise in children. ${ }^{1}$ There is a slight male predominance. The most common presenting symptoms are hematuria and / or nonspecific flank pain which may be attributable to either torsion or intussusception of the polyp or intermittent obstruction. Although the exact etiology of fibroepithelial polyps is uncertain, some have suggested both congenital factors in children and chronic inflammation in adults. ${ }^{1}$

Address for correspondence Dr. Zhen J. Wang, Abdominal Imaging,University of California San Francisco, Box 0628, 505 Parnassus Avenue, San Francisco, CA 94143-0628 Tel: 415-353-1821 Fax: 415-476-0616 Email: jane.wang @ radiology.ucsf.edu. 
Ureteral fibroepithelial polyps vary in size, and can measure up to $12 \mathrm{~cm}$ in greatest dimension. Histologically, fibroepithelial polyps are of mesodermal origins, and are characterized by a loose vascular fibrous stroma with overlying benign transitional epithelium. Despite the benign nature, the majority of fibroepithelial polyps reported in the literature were discovered at the time of nephroureterectomy for a presumed ureteral malignancy. ${ }^{2}$ Although fibroepithelial polyps are themselves benign lesions, cases of coexistent transitional cell carcinoma have been reported. ${ }^{3}$ Bellin et al has also reported a case of an ureteral fibroepithelial polyp with progressive growth documented on serial CT examination. ${ }^{4}$

In general, differential considerations for ureteral lesions include malignant lesions such as transitional cell carcinoma, rare benign mesenchymal tumors, and non-neoplastic etiologies including blood clots, sloughed papillae, fungus ball, or rare parasitic infection. There have been few reports of ureteral fibroepithelial polyps detected on ultrasound. Liddell et al described a case of fibroepithelial polyp of the proximal ureter in a child presenting as a mildly echogenic structure with polypoid projections extended into the right renal pelvis. ${ }^{5}$ In our case, the real-time cine mode imaging and the excellent resolution on ultrasound allowed for the correct preoperative diagnosis of a distal ureteral fibroepithelial polyp in an adult. On ultrasound, the lesion was elongated and smoothly marginated with a vermiform appearance. Cine mode imaging showed that the lesion was mobile and protruded from the right distal ureter into the urinary bladder in real time, a feature not expected for malignancy which is usually fixed in place. The availability of Doppler imaging confirmed a solid mass in this case, therefore excluding entities such as blood clots, sloughed papillae or fungus ball, which may occasional present as a mobile ureteral lesion. CT urogram also showed similar findings of an elongated ureteral lesion with a corkscrew appearance suggestive of a benign fibroepithelial polyp. CT may have an advantage over ultrasound in the diagnosis when the ureteral polyp is entirely located within a non-dilated ureter. In summary, either the sonographic features of a vermiform and mobile solid ureteral mass or the $\mathrm{CT}$ features of an elongated ureteral lesion with a corkscrew appearance should strongly suggest the diagnosis of a fibroepithelial polyp.

The treatment of choice for a fibroepithelial polyp is complete excision. Accurate preoperative diagnosis of this entity is important as it will direct the treatment towards less invasive endoscopic resection rather than more radical surgery such as nephroureterectomy. ${ }^{2,} 6$

\section{References}

1. Bolton D, Stoller ML, Irby P 3rd. Fibroepithelial ureteral polyps and urolithiasis. Urology 1994;44:582-587. [PubMed: 7941202]

2. Faerber GJ, Ahmed MM, Marcovich R, Crisco CP, Belville WD. Contemporary diagnosis and treatment of fibroepithelial ureteral polyp. J Endourol 1997;11:349-351. [PubMed: 9355952]

3. Zervas A, Rassidakis G, Nakopoulou L, Mitropoulos D, Dimopoulos C. Transitional cell carcinoma arising from a fibroepithelial ureteral polyp in a patient with duplicated upper urinary tract. J Urol 1997;157:2252-2253. [PubMed: 9146635]

4. Bellin MF, Springer O, Mourey-Gerosa I, et al. CT diagnosis of ureteral fibroepithelial polyps. Eur Radiol 2002;12:125-128. [PubMed: 11868087]

5. Liddell RM, Weinberger E, Schofield DE, Pelman RS. Fibroepithelial polyp of the ureter in a child. AJR Am J Roentgenol 1991;157:1273-1274. [PubMed: 1950880]

6. Yagi S, Kawano Y, Gotanda T, et al. Endoscopic treatment of a long fibroepithelial ureteral polyp. Int J Urol 2001;8:467-469. [PubMed: 11555017] 
A

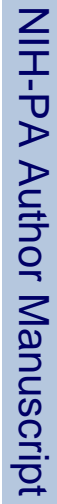

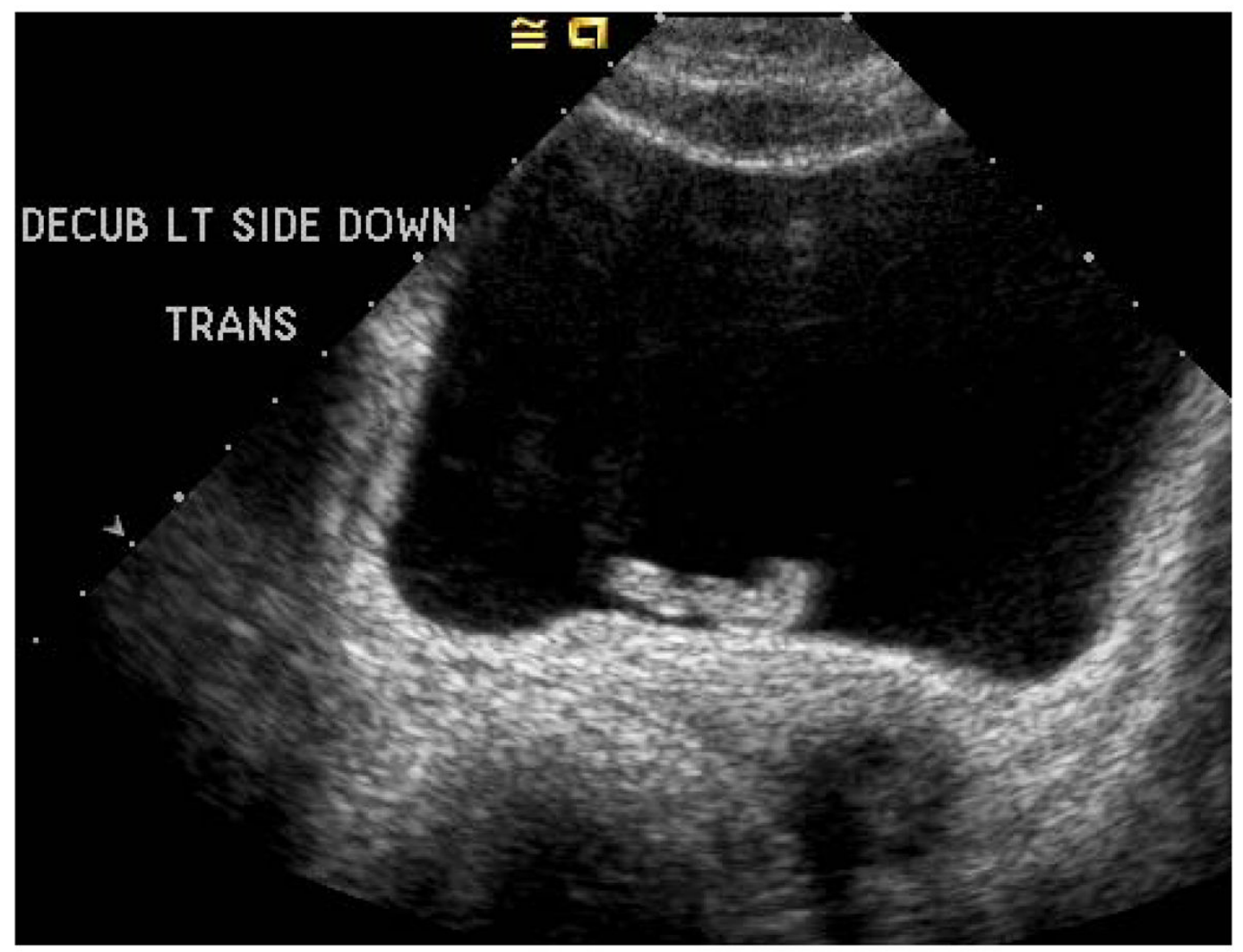

B.

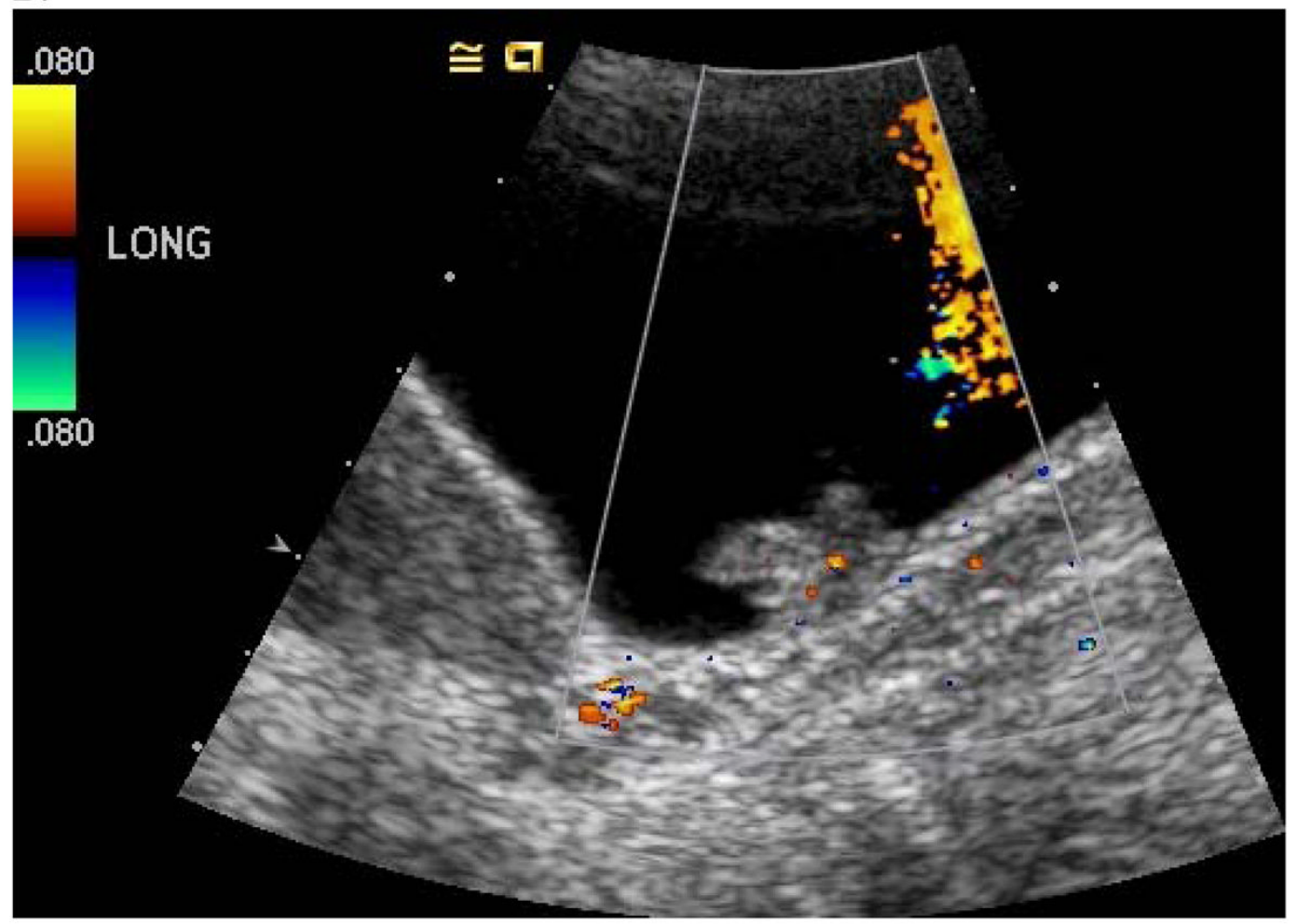

J Ultrasound Med. Author manuscript; available in PMC 2009 September 17. 
Figure 1.

Transverse grayscale image (A) and longitudinal color Doppler image (B) through the pelvis show a vermiform solid mass which appeared to arise from the right distal ureter and protrude into the urinary bladder, consistent with a ureteral fibroepithelial polyp. 


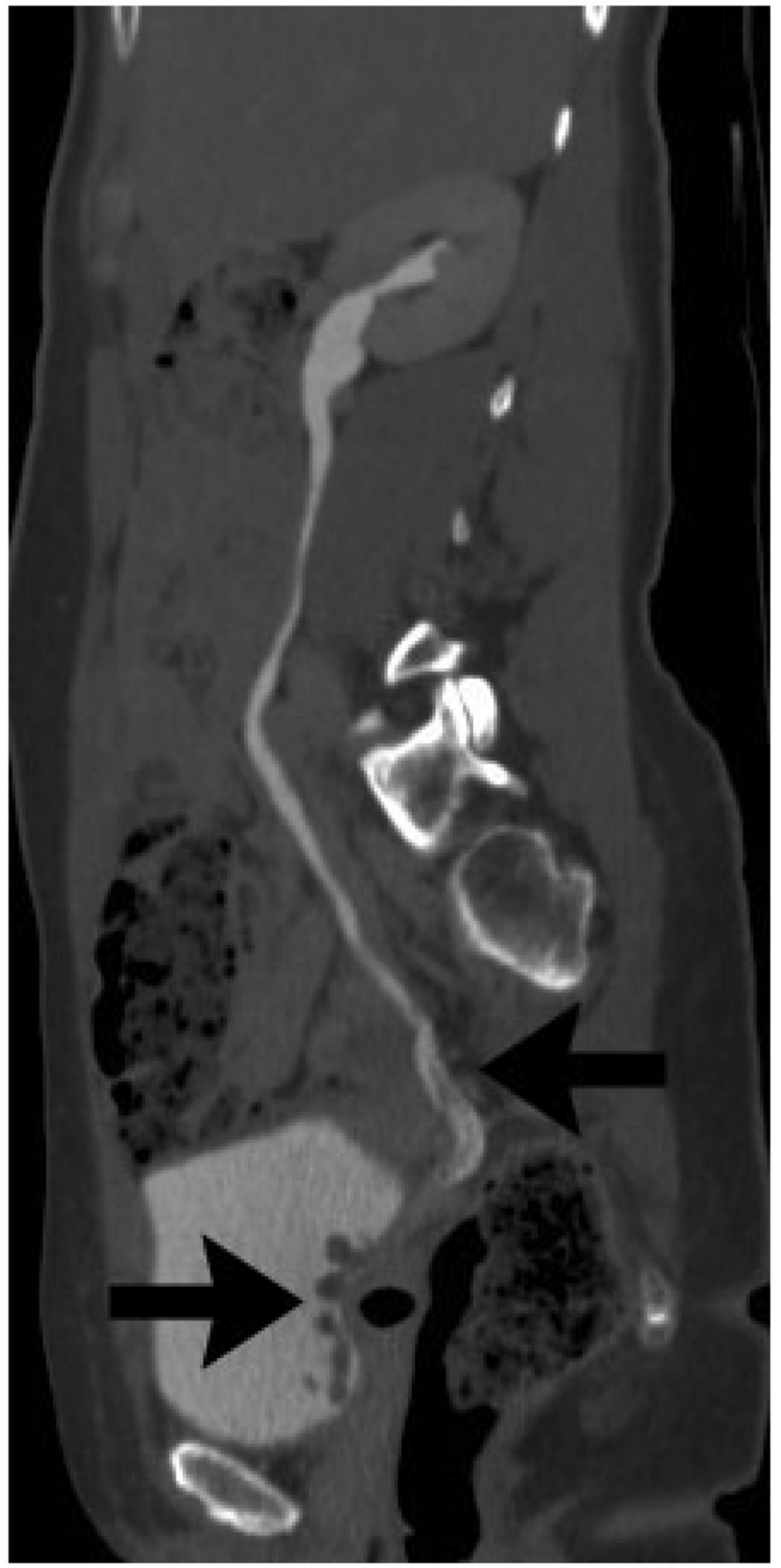

Figure 2.

Sagittal reformatted image from a CT urogram shows an elongated filling defect with a corkscrew appearance within the distal right ureter extending into the urinary bladder (arrows), consistent with a ureteral fibroepithelial polyp. 


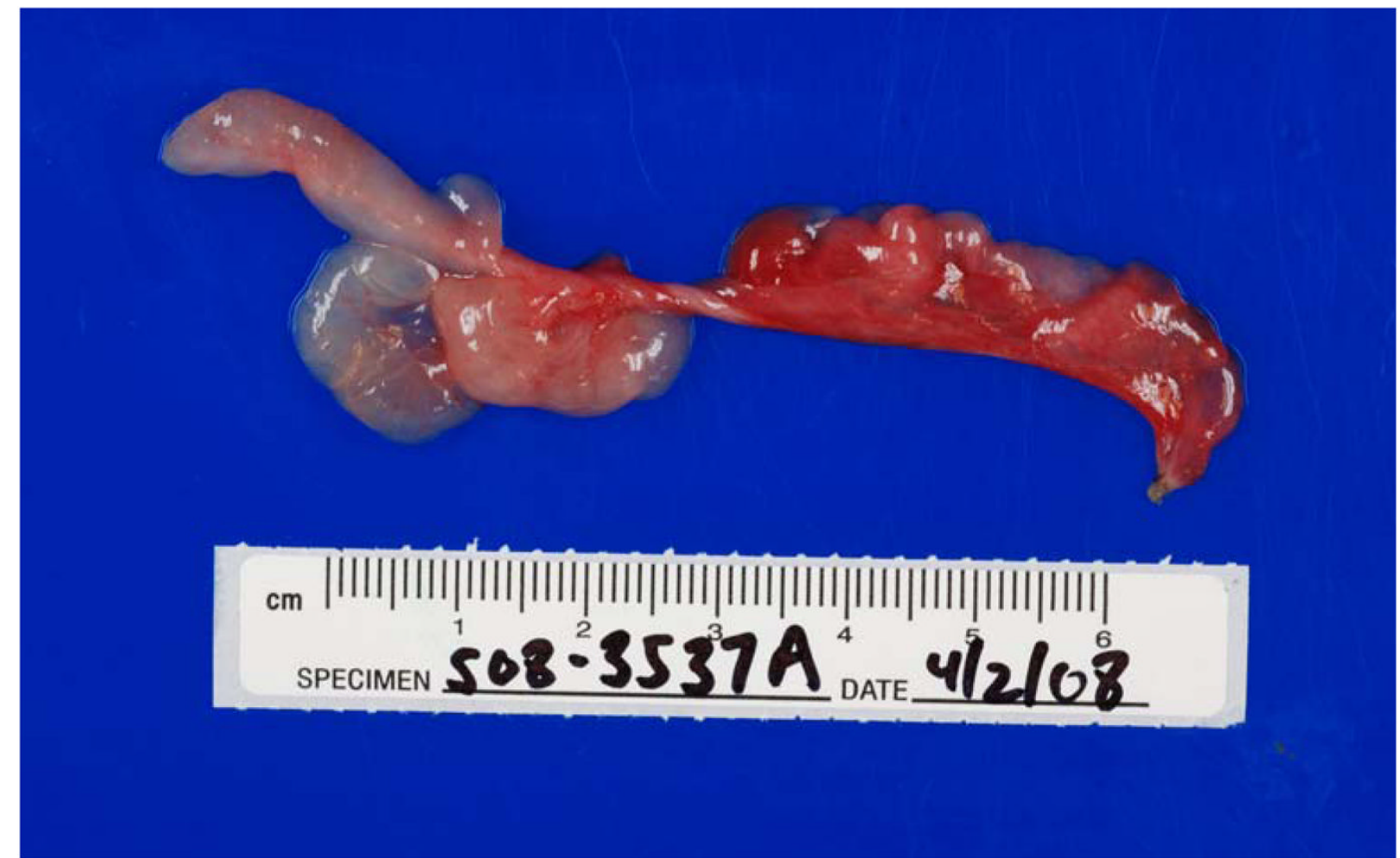

Figure 3.

Ureteroscopically excised right distal ureteral fibroepithelial polyp. 